\title{
A novel approach for modeling bubbling gas-solid fluidized beds
}

Javier Villa Briongos*, Sergio Sanchéz-Delgado, Antonio Acosta-Iborra, Domingo Santana Universidad Carlos III de Madrid. Escuela Politécnica Superior. Departamento de Ingeniería Térmica y de Fluidos, Avenida de la Universidad 30, 28911, Leganés (Madrid)

*Correspondence to the author, e-mail: jvilla@ing.uc3m.es

$†$ This work is dedicated to the memory of Dr. Andrés Cabanillas (CIEMAT) who collaborated during the bench scale combustor measurements. 


\begin{abstract}
A phenomenological discrete bubble model is proposed to help in the design and dynamic diagnosis of bubbling fluidized beds. An activation region mechanism is presented for bubble formation, making it possible to model large beds in a timely manner. The bubbles are modelled as spherical-cap discrete elements that rise through the emulsion phase that is considered as a continuum. The model accounts for the simultaneous interaction of neighbouring bubbles by including the trailing effects due to the wake acceleration force. The coalescence process is not irreversible and therefore, the coalescing bubble pair is free to interact with other rising bubbles originating the splitting phenomena. To validate the model, the simulated dynamics are compared to both experimental and literature data. Time, frequency and state space analysis are complementarily used with a multiresolution approach based on the empirical method of decomposition, EMD, to explore the different dynamic scales appearing in both the simulated time series and those obtained from experimental runs. It is concluded that the proposed model matches the main features of bubble dynamics being a useful tool to aid in the design and dynamic diagnosis of those systems.
\end{abstract}

Keywords: Fluidization, Multiscale modeling, Bubble phenomena; Chaos; Multiresolution analysis. 


\section{Introduction}

Among the huge number of industrial applications of bubbling gas-solid fluidized beds, FB's, those related to their use in energy conversion have recently gained attention due to the current energy policies. Thus, bubbling gas-solid FB's are broadly applied in thermochemical energy conversion processes such as combustion and gasification. The fluidization process offers a high heat transfer rate, good gas-solid mixing and solid handling, and provides a uniform and controllable temperature. Moreover, its ability to process low grade-fuels with low pollutant emission makes the use of bubbling FB's a very promising technology for the necessary valorization of biomass and wastes in energy conversion processes (Jonhsson, 2007). However, when dealing with biomass fluidized bed processes, for instance, the high complexity characterizing conventional gas-solid FB's dynamics increases due to the limited research reported on biomass fluidization hydrodynamics. According to that, recently it has been pointed out the necessity of improving the characterization of biomass fluidization hydrodynamics to understand the influence of the biomass particles on the fluidization phenomena (Cui and Grace, 2007).

The characterization of gas-solid FB dynamics is currently addressed by monitoring the local time evolution of some variables such as pressure, capacitance, temperature, etc. (Werther, 1999). Moreover, some global techniques addressed to characterize the overall fluidized bed dynamics have been also reported (Briongos and Guardiola, 2003; Dyakowski et al. 2000; Briongos et al. 2006a; Van Ommen and Muddle, 2007). Subsequently, in order to elucidate the dynamical processes occurring within the FB system, the time dependent behaviour of the measured signals is often analyzed by time and frequency domain analysis (Jonhsson et al. 2000). However, due to the inherent nonlinear features of gas-solid FB dynamics, a non linear approach including a multiscale analysis and tools derived from the deterministic chaos theory, can be reliably applied complementarily to the time and frequency analysis methods to account for those nonlinear interactions (Briongos et al. 2006b). Thus, dynamical aspects characteristics of gas-solid fluidization, are identified by establishing a direct relation between the physical phenomena driving the dynamics, and the measured signals. Nevertheless, when dealing with complex processes such as the fluidization of biomass particles, previous literature often neglects critical dynamical aspects related to biomass particle properties, ash features or endogenous bubble generation (Fiorentino et al., 1997), which makes even harder the understanding of the physical phenomena behind the measured signals. According to that, in order to improve the characterization of the dynamics of bubbling gas-solid FB's, it is requisite to understand both the dynamics behind the measured signals and the way the different signal properties obtained from the digital signal processing are related to the physical phenomena occurring within the FB's. Thus, CFD models of gas-solid systems are often used to extract information useful to guide design and operation of fluidized beds (Gidaspow, 1994). During the last decade due to the shocking increase of the computer capabilities there are a huge number of literature dealing with gas-solid FB modelling, the models can be divided into two groups, Eulerian-Eulerian models, which consider the gas and solid phases as interpenetrating continua (Ding and Gidaspow, 1991; van Wachem et al. 2001; Lindborg at al., 2007), and Eulerian-Lagrange models that coupling a Lagrangian description of the particle dynamics with a continuum description of the gas-phase (Deen et al., 2007).

Either the continuum or the discrete element method, DEM, approaches involve a detailed description of the flow, in one hand, the discrete particle model approach (Tsuji 
et al. 2008), DPM, try to describe the large-scale dynamics appearing in fluidized beds by direct modelling of the particle-particle and gas-particle interactions. Though DPM is a very powerful tool to study the details of the multiscale flow structure characterizing gas-solid FB's, however, it is well known that the success of the DPM models is not guaranteed since a detailed description of particle dynamics is not always possible (Deen et al., 2007), and some correlations between the small and large-scale dynamics can be missed. Besides, the vast computational effort needed to model a large number of particles limits its use to model large FB units. On the other hand, the continuum approach, though it is also time-consuming, it can be used fairly well for scale-up design studies, however, the information provided on dynamic characteristics is very limited, and its solution strongly depends on empirical closure relationships. Moreover, few works in the literature validate the simulated behaviour of the FB using the same information that is currently measured during FB monitoring such as pressure fluctuation signals (van Wachem et al., 1999). Furthermore, due to the detailed description of FB dynamics, in the end both the continuum and the DPM approaches become as complex to characterize as the experimental unit, making it difficult to identify and therefore, to establish a direct connection between the bed dynamics phenomena and the measured signals. To simplify the problem, some simpler models have been proposed in literature to diminish the number of variables by studying either the particle or the bubble dynamics (Van den Bleek and Schouten, 1993; Daw and Hallow, 1992), to later extrapolate the information provided during the simulation to the observed experimental behaviour. According to that, two recent different approaches addressed the DBM of FB's. They have shown to be useful for modelling global dynamics of large scale FB's. Thus, the Dynamic Interacting Bubble Simulation model, DIBS, proposed in Pannala et al. (2004), which accounts for the simultaneous interactions of individual bubbles and have been successfully applied for reacting FB's. However, the fact that both the bubble rise velocity and the trailing effect between rising bubbles are based on empirical correlations make the general use of DIBS difficult for modelling bubbling FB's. In contrast to the DIBS model, the DBM reported in Bokkers et al. (2006), fully accounts for the two way coupling between the emulsion phase and the rising bubbles, which are considered as discrete spherical elements, that rise according to the second law of motion, moreover the model includes the bubblebubble and bubble-wall interaction to model the coalescence process. Nevertheless, the force resulting from bubble to bubble interaction due to wake effect is ignored, and the inclusion of the Eulerian solution for the dense phase increases the computational cost.

In this paper a DBM approach is presented to help the understanding of the dynamics behind the measured signals and consequently, to improve the existing monitoring methods by relating the different signal parameters obtained from the time series analysis to the physical phenomena occurring within FB's. In contrast to previous discrete bubble models reported in literature, a wake acceleration force is proposed to account for the bubble trailing interaction. The force balance is based on the pressure recovery in the wake below a spherical cap bubble (Davidson and Harrison, 1963). Moreover, a novel activation region mechanism is proposed to explain the multi-orifice bubble generation. Furthermore, the bubbles are considered as discrete spherical caps, and are tracked individually by integrating the equations derived from the second law of motion. The model uses a direct implementation of the bubble coalescence and does not make any prior assumption on bubble encounter frequency. 


\section{The discrete bubble model}

\section{Model basis}

The proposed model is a discrete phenomenological approach where bubbles are modelled as spherical-cap discrete elements that rise through the emulsion phase that is considered as a continuum. The modified two phase theory, in which it is considered that not all the excess gas, $\mathrm{U}_{\mathrm{e}}=\mathrm{U}_{0}-\mathrm{U}_{m f}$, passes through the bed as observable bubbles, is used to estimate the visual bubble flow. Accordingly, the visible bubble flow, $V_{b}$, is given by the well known relationship:

$V_{b}=\psi U_{e} A_{b e d}$

Where $\psi$ is the dimensionless ratio between the observed bubble flow and the excess flow from the two phase theory (Kunii and Levenspiel, 1991), and it is estimated according to the correlation proposed in Johnsson et al. (1991). Furthermore, the bubbles are dynamically coupled to its closest leading neighbour through a wake acceleration force that accounts for the bubble trailing interaction. The bubble coalescence is modelled including a shrinking/growing mechanism that gradually increases or decreases the size of the coalescing bubble pair. Moreover, bubble formation at the distributor plate has been modelled according to an activation region mechanism to provide different bubble patterns as reported in Whitehead (1985).

\section{Bubble generation}

Bubble formation phenomena will influence the distributor performance as well as the final bubble pattern developed within the bed, however, whereas bubble generation at a single orifice has been widely studied, and some models have been proposed for computing the bubble volume at the detachment (Davidson and Harrison, 1963; Caram and Hsu, 1986; Vakhoshouri and Grace, 2008). To the authors' best knowledge, the information reported in literature regarding the multi-orifice bubbling formation in gassolid fluidized beds is rather scarce (Leung, 1971; Rees et al., 2006; Müller et al., 2009). In principle, the natural approach for multi-orifice bubbling generation would be to address the case of multiple orifices as an extension of single orifice bubble formation. However, several factors such as gas leakage and the rapid formation of doublets and triplets by coalescence of the emerging bubbles, make the previous discrete bubble models appearing in the literature use instead empirical correlation to estimate the initial bubble size. Besides, other critical aspects of bubble formation, such as the bubble injection frequency and the subsequent injection pattern, have been often left out of the discussion. It is clear therefore, that the bubble generation mechanism will play a major role which must be addressed with caution to satisfactorily explain the observed performance of distributors in FB's.

In contrast to previous works, in this paper, an extension of the single orifice model proposed in Davidson and Harrison (1963) will be used as a single-orifice departure model to further extend the bubble generation to a multi-orifice distributor plate. According to that, at a superficial velocity greater than $U_{m f}$, the bubble volume formed at the orifice is given by:

$V_{0}=\frac{1.138 G^{1.2}}{g^{0.6}}$,

where $\mathrm{G}$ accounts for the bubble flow per hole that within the proposed model reads as:

$G=\frac{V_{b}}{N_{o r}}=\frac{\psi U_{e} A_{b e d}}{N_{o r}}$,

Next, the bubble frequency above the orifice can be estimated according to: 
$f_{b, o r}=\frac{G}{V_{0}}$,

Once both the bubble detachment volume and the bubble orifice frequency are estimated, the extension of the bubble generation to the multi-orifice system is not trivial. As stated above, little is known about multi-orifice bubbling, and previous reported works skip out of the discussion regarding the bubble injection patterns and the bubble generation frequency. Traditionally, due to the existing analogies between gasliquid and gas-solid systems, the research carried on for gas-liquid mixtures is currently used to get knowledge on gas-solid fluidized beds and vice versa. According to that, it can be argued that the few examples found in literature on multi-orifice bubble generation dealing with uniform gas-liquid mixtures might be used to devise a multiorifice mechanism for fluidized beds (Ruzicka et al, 1999). Thus, those studies explore how design and operation conditions such as pressure and plenum volume, orifice spacing, and liquid depth affect bubble size as well as bubbling synchronicity (Xiao and Tan, 2003). However, they are based on single orifice models (Zhang and Tan, 2000), therefore, the gas flow through each orifice is determined from orifice equations, the estimated bubble volume is larger as pressure drop across the orifice increases. As a consequence, the higher the pressure drop, the larger the bubble will be. Moreover, the average gas flow thorough each orifice is obtained by dividing the gas flow rate by the total number of orifices, which leads to a decrease of the initial bubble size as increasing the orifice number. Apparently that approach holds for submerged single orifices in fluidized beds (Vakhsouri and Grace, 2008), however, when extrapolating to multiorifice generation, the direct implementation of that approach would conclude that the initial bubble size increases as the open area ratio decreases, which is contrary to the experimental evidence on distributor performance that shows how bubble size increases when the open area is increased. In fact, it is well known that pressure drop across distributors indeed influences the bubble regime at low fluidization velocities (Svensson et al. 1996a). Thus, for instance, it has been reported that a large pressure drop should lead to multiple bubble regime, which is characterized by many relatively small bubbles which are well distributed over the cross-section of the bed, whereas for small pressure drops, single bubble regime might appear. In that regime the bubbles are larger in size and unevenly distributed over the cross-section. Then, how to reconcile both the bubble generation mechanism and the distributor performance?

\section{The activation region mechanism}

The results reported on uniform gas-liquid systems (Ruzicka et al, 1999; Xiao and Tan, 2003), reveal that bubbling synchronicity is strongly influenced by the spacing and arrangement of the orifices, liquid depth and gas flow rate, however, no explicit model is provided to account for that influence, which makes it very difficult to devise how the bubble synchronization can take place and consequently, nothing can be concluded about what to model; i.e. doublets, triplets, etc. However, those studies also report the fact that the orifices active in one part of the distributor enhanced the bubble generation within their nearest distributor region, making the orifices which belong to other distributor regions passive for bubble formation. Moreover, recently it has been reported that in the case of multi-orifice distributors, for $U_{0}>U_{m f}$ conditions, the region near the distributor plate exhibits permanent jets (Müller et al. 2009), and the bubble detachment occurs above that region. That information serves here to propose the activation region mechanism, where the multi-orifice plate is seen as a discrete source of information where the bubbles are the dynamical "message" to be transmitted, that sequence of bubbles follows a certain generation rate and injection pattern (Figure 1), the injection 
pattern establishes the probability of bubble formation of the different distributor regions. Through this paper, the injection pattern shown on Figure 1d has been used to match the behavior of distributor systems Type 1, as described in Whitehead (1985) (Appendix A). With regard to the generation rate it is assumed that in the same way in which the single orifice is characterized by a bubble injection frequency, similarly the multi-orifice distributor is characterized by an overall bubble generation frequency, $f_{b}$. The overall bubbling frequency defines a rate of region activation, according to that, bubbles of size $v_{b}$ given by:

$v_{b}=\frac{V_{b}}{f_{b}}$

The bubbles will be generated at different regions on the distributor plate at a rate defined by $f_{b}$. Consequently, it is assumed that the resulting bubble size is the consequence of the coalescence of bubbles generated at neighbor orifices. Moreover the bubble appears above the jet permanent area, JR. Figure 2 shows a picture of an experimental 2D fluidized bed system operating at bubbling conditions with a multiorifice distributor, which serves to illustrate the idea behind the activation region mechanism. Thus within the proposed model, the generated bubbles are the result of the interaction of neighbor orifices and will detach within the activation region, AR (Figure 2 ). The use of that approach makes the computational cost decrease significantly, which is an enormous advantage of the proposed methodology, and brings the opportunity to model large scale beds in a common desktop computer in a timely manner. Moreover, to allow for this region activation mechanism to occur, the orifice frequency is assumed to be non-uniformly distributed over time. Finally, in order to estimate $f_{b}$, two different approaches are presented below, the synchronous and the asynchronous models.

\section{The synchronous model}

This generation approach does not include the distributor performance. Instead, the hypothesis underlying the synch-approach, assumes an ideal situation where the bubble injection occurs simultaneously from all orifices. Consequently, the region activation rate will be characterized by the bubble frequency above the orifice resulting from the orifice departure model. Therefore, under those conditions the overall bubble generation frequency, $f_{b}$, would be $f_{b}=f_{b, o r}$. However, due to the fact that the bubble generation frequency is directly derived from the orifice theory, when the design of the virtual distributor used in the model matches the real distributor, the model predicts erroneously the expected dynamical behavior of the bed unit.

Nevertheless, a generation approach where the effect of the distributor performance is not included can still be useful for dynamic diagnosis and dynamical matching. Thus, just by ignoring the distributor, the overall generation frequencies can be arbitrarily varied until matching the dynamical features experimentally observed in the FB under diagnosis i.e. through pressure fluctuation measurement.

According to that it is clear that in order to avoid the limitation of the synch-model for designing purposes, the distributor performance needs to be taken into account.

\section{The asynchronous model}

To facilitate the use of the activation region mechanism for designing purposes, the asynch-approach assumes that the distributor performance will produce a deviation on the predicted $f_{b, o r}$ by the previous synch-model. Consequently, the overall bubble generation frequency will be given as:

$f_{b}=\phi \cdot f_{b, o r}$ 
The challenge now is to estimate the deviation coefficient, $\phi$, which should account for the distributor performance. It is clear that the operation of a distributor is closely related to its design which will influence both the mixing of solids, and the fluidization quality. A measure of the stirring effect of a distributor is the factor $\alpha_{j}$, (Kunii and Levenspiel, 1991).

$\alpha_{j}=\frac{\rho_{g} u_{o r}^{2} / 2}{\Delta P_{b}}$

The factor $\alpha_{j}$ gives the relation between the kinetic energy of the orifice jets and the resistance of the bed, measured by the bed pressure drop, $\Delta P_{b} . u_{o r}$ is the gas velocity through the orifice that, as it is well known, is closely related to the pressure drop across the distributor, $\Delta P_{d}$. Thus, as $\Delta P_{d}$ increases, $u_{o r}$ increases and consequently, the stirring effect of the distributor, $\alpha_{j}$, increases. It has been reported that when $\alpha_{j}>1$ the stirring capacity of the distributor is mostly due to the jets formed at the orifices, whereas for $0.09<\alpha_{j}<1$ the stirring capacity is due to both jets and bubbles formed at the distributor and finally, for $\alpha_{j}<1$ the jets do not contribute much to the bed stirring and bubbles should do the job.

According to that, the stirring capacity of the distributor, $\alpha_{D}$, should have at least two contributions: the contribution due to jet stirring, $\alpha_{j}$, and the mixing promoted by the bubbles formed at the distributor, $\alpha_{b}$. Thus, the methodology followed to estimate de deviation coefficient consists on computing the stirring capacity of the active area appearing at the distributor, $\alpha_{D a}$, and later to extend that capacity to the rest of the distributor.

Once $\alpha_{j}$ have been calculated according to Eq. 6, by analogy to the jet stirring, the factor $\alpha_{b}$ accounts for the relation between the kinetic energy due to bubbles formed at the distributor plate and the resistance of the bed.

$\alpha_{b}=\frac{\rho_{e} u_{b, o r}^{2} / 2}{\Delta P_{b}}$,

Where $u_{b, o r}$ is the initial bubble velocity and it is estimated from the results of the synchmodel as:

$u_{b, o r}=\frac{d_{b 0}}{2 t_{d}}$,

Where $d_{b 0}$ includes de wake fraction, $f_{w}$, as:

$d_{b o}=\left(\left(1+f_{w}\right) \cdot v_{b} 6 / \pi\right)^{1 / 3}$,

$f_{w}$ it is estimated according to Hoffmann et al. (1993) and $t_{d}$ is the detachment time given by:

$t_{d}=\frac{1}{g^{3 / 5}}\left(\frac{6 V_{b}}{\pi}\right)^{1 / 5}$,

Finally, the stirring capacity of the active area region of the distributor, $\alpha_{D a}$, is given as the weighted mean of the two components:

$\alpha_{D a}=w_{j} \alpha_{j}+w_{b} \alpha_{b}$,

Where the weights $w_{j}$ and $w_{b}$ are estimated as a first approximation as:

$w_{i}=\frac{\alpha_{i}}{\alpha_{j}+\alpha_{b}}, \quad i=j, b$, 
The use of the weighted mean allows the jet component to dominate at high $\Delta P_{d}$, whereas the bubble component dominates at low $\Delta P_{d}$, being consistent with the observed experimental behavior. Finally the deviation coefficient is computed by scaling the stirring capacity obtained from Eq. 11 to the overall distributor section.

$\phi=\frac{\alpha_{D a}}{f_{a}}$,

Where $f_{a}$ is the fraction of active area relative to the total distributor area that is estimated as:

$f_{a}=\frac{\text { area of the active region }}{\text { bed area }}=\frac{(\pi / 4) d_{b 0}^{2}}{A_{b e d}}$,

\section{Bubble rise}

Following the results reported in Briongos et al. (2007), the proposed bubble model assumes that the bubbles are the driver of bubbling fluidized beds dynamics, which is in agreement with previous approaches reported in literature (Daw and Halow 1992; Pannala et al., 2004). In contrast to those previous models where the bubbles are considered as spherical elements, in the present approach, the bubbles are considered as spherical caps which rise according to their size and local condition. Moreover, instead of computing the bubble trajectory by integrating the bubble velocity in time from empirical correlations, the bubbles are tracked individually according to Bokkers et al. (2006), where the virtual mass force has been modified to account for the spherical cap (Kendoush, 2003).

$$
\frac{d}{d t}\left[\left(m_{b}+m_{v}\right) U_{b}\right]=\left(\rho_{g}+C_{v} \rho_{e}\right) v_{b} \frac{d}{d t} U_{b}=F_{B}+F_{D},
$$

Where $F_{B}$ is the effective buoyancy force and $F_{D}$ is the drag force acting on the bubble, which are given by:

$$
\begin{aligned}
& F_{g}=-\frac{\pi d_{b}^{3}}{6} \rho_{g} g, \\
& F_{b}=\frac{\pi d_{b}^{3}}{6}\left(\rho_{g}+(1-\varepsilon) \rho_{p}\right) g, \\
& F_{B}=F_{b}+F_{g}=\left(\frac{\pi d_{b}^{3}}{6}\right)\left(\rho_{p}-\rho_{g}\right) g(1-\varepsilon), \\
& F_{D}=-\frac{1}{2} C_{D} \rho_{e} \frac{\pi}{4} d_{b}^{2} U_{b}^{2},
\end{aligned}
$$

According to Bokkers et al. (2006), the drag coefficient for single bubble rising is computed from the steady state force balance giving:

$$
C_{D}=\frac{4}{3} \frac{\left(\rho_{e}-\rho_{g}\right)}{\rho_{e}} \frac{d_{b} g}{U_{b}},
$$

Thus, each bubble trajectory along the $\mathrm{Z}$ axis is estimated by integrating the equation 15, whereas the dynamical coupling between rising bubbles that lead to the XY displacement is driven by the wake acceleration force that results from the trailing bubble effect (Figure 3a). 


\section{Trailing bubble effect}

The behaviour of bubbles in fluidized beds has received considerable attention, and expressions for estimating the rising velocities of bubbles have been previously reported (Davidson et al. 1985). Moreover, it is well known that bubbles rise more rapidly when rising in a bubble stream than in isolation due to the process of bubble coalescence (Grace and Harrison, 1969). Thus the wake of the leading bubble accelerates the trailing bubble before the coalescence process takes place. Accordingly, it is clear that in order to simulate the observed bed behaviour, the trailing effect should be taken into account to model the behaviour of an interacting stream of bubbles. In previous works, the dynamical coupling between neighbours bubbles was either neglected (Bokkers et al., 2006) or described through empirical relationships (Daw and Halow 1992; Pannala et al., 2004). In contrast, in this paper an interacting bubble model is proposed to describe the trailing bubble effect of the leading bubbles. The model is based on the pressure recovery in the wake below a spherical cap reported in Davidson and Harrison (1963), and on the idea suggested by Clift and Grace (1971) of adding to the isolated bubble velocity a component related to the particulate phase. The hypothesis is that the pressure drop originated by the wake of the leading bubble will cause acceleration of the corresponding trailing bubbles by means of a void propagation mechanism, thus the increase of velocity due to the wake acceleration force equals the velocity that the dense phase would have at the position of the trailing bubble.

According to that, the pressure recovery is obtained by applying Bernoulli's theorem to the system shown in Figure 3b (Davidson and Harrison, 1963).

$p_{R}=\rho_{g} g h-\frac{1}{2} \rho_{g} U_{b}^{2}$,

Later Bernoulli's theorem is applied again to estimate the void propagation velocity which corresponds to the increase of bubble velocity due to the wake acceleration force (Figure 3c):

$U_{\text {void }}=\sqrt{\frac{2 p_{R}}{\rho_{e}}}$

The bubble velocity of the trailing bubble is finally given as the sum of its isolation velocity plus the void propagation velocity:

$U_{T b}=U_{i b}+U_{\text {void }}$

\section{Bubble coalescence}

The coalescence of bubbles is modelled following the approach reported in Daw and Halow (1992). Consequently, during the coalescence there is a net gas exchange between the lower and upper bubbles that governs the process. However, instead of defining the gas exchange rate, $Q_{c}$, as a function of bubble rise velocities, the through flow velocity across any plane through the bubble derived from the alternative analysis presented in Lockett et al. (1967) is used as constant gas transfer rate during the coalescence, being therefore as:

$Q_{c}=3 U_{m f} \frac{\pi}{4} d_{L b}^{2}$,

Moreover, it is worth mentioning that neither the upper bubble nor the lower bubble are bound to the coalescence process; they are still free to interact with any neighbour bubble according to the phenomenological assumption presented below that ruled the interactions. That fact makes possible the splitting of the coalescing bubble pair to 
appear, giving rise to a final bubble size distribution resulting from coalescing and splitting processes.

\section{Other phenomenological key assumptions}

I. Bubbles grow only by coalescence.

II. Wall effects are not taken into account.

III. A bubble is a trailing bubble if it lies within the projected horizontal area defined by twice the diameter of its closest leading bubble and, if their center-to-center distance is less or equal than that of four times the leading bubble radii (Clift and Grace, 1971).

IV. In order for the coalescence processes to take place, the trajectory followed by the nose of the lower bubble should fall within the overlap region defined by one times the diameter of the upper bubble.

V. When the nose of the lower bubble enters the wake of the leading bubble (Hoffmann et al., 1993), the coalescence process begins by shrinkage of the lower bubble and subsequent increase of the upper bubble. Coalescence will continue until the complete depletion of the lower bubble or until the splitting of the coalescing pair as a result of the interaction with neighbour rising bubbles.

VI. Bubbles will exit the bed when their centers reach the bed surface.

VII. Either when a bubble leaves the bed or when a bubble disappears as a result of the coalescence process, the total number of bubbles in the bed is reduced by 1 .

\section{Experimental Design}

This paper addresses the reliability of the proposed region activation model to simulate the dynamics of bubbling fluidized bed having either perforated plate or Tuyere type distributors (Table 1), and operating with B-Geldart particles. Accordingly, in order to validate the proposed approach, the dynamic characteristics of the simulated system are compared with both experimental and literature pressure fluctuation data. Thus the cold rig used in both Johnsson et al. (2000) and in Svensson et al. (1996a, 1996b) to study the influence of pressure drop across the distributor on bottom bed regimes in CFB's. It serves here for testing the reliability of the region activation model for designing purposes, and dynamic diagnosis matching of FB's having multiorifice distributors. Moreover, as an example of the use of the proposed model for Tuyere type systems, experimental data collected from a bench-scale combustor operating at ambient temperature are presented for model validation under current bubbling operating conditions. Most of the model settings used through the simulation as well as the fluidization unit characteristics are shown in Table 1.

Pressure fluctuation measurement

Multi-orifice distributor system

The experimental time series used during the validation of the multi-orifice distributor model have been taken from Johnsson et al. (2000), accordingly, the pressure time series were collected by means of Kistler Type 7261 transducer placed at $0.2 \mathrm{~m}$ height and the sampling frequency used was $400 \mathrm{~Hz}$. A more detailed description of the measurement acquisition system can be found within the mentioned contribution.

Tuyere type system

The pressure fluctuations were measured by means of two pressure gauges PR3110 (Ellison Sensors) placed at two different positions: position $1(0.1 \mathrm{~m}$ over the distributor) and position 2 (0.2 m near the bed surface), and connected to a PCI 6023E I/O board (National Instruments). The sampling frequency used was $200 \mathrm{~Hz}$. 
The Davidson model (Davidson and Harrison, 1963) is used to estimate the pressure time series. Thus, the pressure due to a single bubble passing a probe is estimated as:

$$
\begin{aligned}
& P_{b}=\rho_{s} g\left(1-\varepsilon_{m f}\right)\left(r-\frac{R_{b}^{3}}{r^{2}}\right) \cos \theta, \quad r \geq R_{b} \\
& P_{b}=\rho_{s} g\left(1-\varepsilon_{m f}\right) r \cos \theta, \quad r<R_{b}
\end{aligned}
$$

Where $R_{b}$ is the bubble radius and $r$ is the distance from the bubble center to the pressure probe. Later, the simulated pressure time series caused by all the bubbles rising through the bed is estimated as the sum total of $P_{b}$ :

$$
P_{m}=\sum P_{b}
$$

Besides the simulated time series, other relevant information such as the bed height fluctuation, void fraction, bubble size and angle of gyration (Pannala et al., 2004) is collected to help the dynamic diagnosis.

\section{Results}

\section{Perforated plate distributor}

The validation of the proposed approach for designing and dynamic diagnosis of gassolid fluidized beds having a multiorifice distributor undergoes two steps. First, the influence of the pressure drop across the air distributor is studied by comparison of the simulated data with the results reported in Svensson et al. (1996a), for bottom bed regimes appearing in circulating fluidized beds, CFB's. Later, a detailed dynamical comparison between the dynamics characterizing the simulated time series, and the Kistler pressure fluctuation data reported in Johnsson et al. (2000), is performed to study the different dynamical scales appearing in the model. Thus, frequency domain, stated space analysis, mutual information function, and multi-resolution analysis by applying the Empirical Method of Decomposition, EMD (Briongos et al. 2006b), are complementarily used to perform the comparison between both the simulated and the measured pressure fluctuation data.

The simulated cold rig has a cross-section of $0.12 \times 0.70 \mathrm{~m}$ having five different perforated plates (Table 1), the bed material is Silica sand particles of $320 \mu \mathrm{m}$ and 300 $\mu m$ in size respectively, and the virtual pressure probes are located at $0.05 \mathrm{~m}, 0.15(0.2)$ $\mathrm{m}$. Moreover, other model outputs are the pressure drop fluctuation which is estimated as: $\Delta P=\rho_{p}\left(1-\varepsilon_{f}\right) g h_{f}$, the bed height, $h_{f}$, the void fraccion $\varepsilon_{f}$ and the non-dimensional cross-sectional area of the bubble stream, $A_{r}^{*}$ (Pannala et al., 2004).

\section{The influence of pressure drop across the air distributor}

The Figure $4 \mathrm{a}$ shows the power spectra estimated from the simulated pressure fluctuation time series. It can be seen how the pressure drop of the air distributor, $\Delta p_{\text {dist }}$, influences the fluidization behavior of the bed at low fluidization velocities. Thus, as same as the results reported in Svensson et al. (1996a), large $\Delta p_{\text {dist }}$ (low number of orifices), lead to multiple bubbling regime, MBR, which is characterized for a wide range of relatively high frequencies up to $3 \mathrm{~Hz}$, whereas low $\Delta p_{\text {dist }}$ lead to single bubble regime, SBR, which exhibits a sharp peak below 1Hz. Moreover, the Figures 4b, c show how the pressure drop influences both the bubble pattern and the bubble size distribution promoted within the bed. Consequently, from a visual comparison of the simulated data shown in Figure 4 to the results reported in Svensson et al. (1996a) it might be concluded that the proposed model apparently provides a reliable quantitative and qualitative description of the measured bubbling fluidized bed dynamics. However, 
it is worth to point out that the sampling frequency used in Svensson et al. (1996a) was $20 \mathrm{~Hz}$, which is enough to collect the low frequency dynamical information below $2 \mathrm{~Hz}$ but insufficient to explore the high frequency dynamical regions reported later for the same test rig in Johnsson et al. (2000). According to that, from a detailed dynamical comparison of the simulated data, the results reported in Johnsson et al. (2000) are more suitable since they were measured by using high sensitive pressure transducer, and a sampling frequency of $400 \mathrm{~Hz}$.

Figure 5 shows the power spectrum for both the simulated data and the experimental time series used in Johnsson et al. (2000) for either single, multiple and exploding bubble regimes, ER. It can be observed how the model apparently matches the measured dynamics for SBR, whereas it exhibits clear differences for both the MBR, and for the ER. In contrast to the previous results reported in literature, where MBR was characterized by frequencies up to $3 \mathrm{~Hz}$, the experimental MBR spectrum shown in Figure 5 exhibits frequencies ranging approximately up to $10 \mathrm{~Hz}$. The high sensitivity of the pressure transducer and the larger sampling frequency used, facilitate the collection of more dynamical information within the high frequency region, which is attributed to dense phase dynamical processes, which are mainly due to the dynamic interactions between the bubble and the dense phase. The fact that those interactions are not explicitly modeled, make the simulated pressure signals, which are derived from the bubbles existing within the bed, Eq. 27, carry information ruled by the bubble dynamics, and that they just contain little information about the global bed motion through the effect of bed height fluctuation on bubble rise dynamics (Eq. 18). Consequently, the simulated time series are mainly characterized by low frequency components. Therefore, the direct comparison between the simulated data, and the experimental pressure fluctuation time series for both the MBR and the ER, whose dynamics are strongly influenced by dense phase processes, would be biased due to the strong influence which the high frequency components have on the measured signals. Accordingly, instead of direct comparison, a multi-scale analysis is proposed to use in order to compare the corresponding low frequencies dynamics of both simulated and experimental pressure time series collected at MBR and ER conditions.

The Single Bubble Regime has been identified when the FB operates with a low pressure drop of the air distributor. Moreover, it has been reported that the gas flow apparently exhibits a discontinuous behavior ruled by the bubble dynamics, being therefore, the formation and eruption of large bubbles the dynamical feature which characterizes this regime (Svensson et al. 1996b). According to that, as expected, the Figure 5a shows a strong dynamic similarity between the simulated and the experimental pressure time series, since in essence the proposed model is based on formation, interaction, and eruption of bubbles. Consequently, there is no need to perform a multi-scale approach to validate the model performance for SBR conditions, the direct comparison between the measured signal and the simulated time series suffices to point out the remarkable matching between the compared signals. Accordingly, the frequency domain analysis shown in Figure 5a reveals that both the exploding bubble frequency (low frequency peak), due to bubble dynamics, and the single bubble frequency peak corresponding to the bed natural frequency, match. However, as the mutual information analysis shown in Figure 6 (Fraser and Swinney, 1986), both the simulated and the measured time series have differences within their short-term temporal structure. Thus, the mutual information function of the measured signal exhibits persistence corresponding to the high frequency peak, observed during the frequency domain analysis, where as such persistence appears attenuated within the simulated data. As stated above, the simulated pressure time series lacks information 
concerning dense phase phenomena, and only the bed height fluctuation slightly influences the simulated signal through the void fraction, $\varepsilon$, in Eq. 18. Consequently, the bulk dynamics (Briongos et al. 2006b) is responsible for the differences within the short-term temporal structure between both the measured, and the simulated pressure time series observed in Figure 6.

Complementarily to the frequency domain and mutual information analysis, the state space analysis has been used to validate the model. Thus, Figure 7 shows the principal component analysis and the eigenvalue spectra estimated according to the Broomhead and King method (1986). As expected from the previous analysis, the resulting attractor structure and eigenvalue spectra for both the simulated, and the measured pressure time series are very similar. Moreover, other traditional nonlinear measures such as the correlation dimension, $\mathrm{D}_{2}$ (Grassberger and Procaccia, 1983), and the Kolmogorov entropy per cycle, $\mathrm{K}_{\mathrm{c}}$ (Schouten et al., 1994), shown in Figure 7, are consistent with the expected values. Consequently, the measured pressure time series are characterized by a Kolmogorov entropy lower than the value estimated from the simulated time series, as a consequence of the persistence occurring at short-term scale. Moreover, the high degree of similarity between the reconstructed attractors is confirmed by the same value of the correlation dimension, which, as it is well known, is a measure of spatial homogeneity.

As stated above, a multiscale approach is needed for comparison when operating at MBR. Consequently, in order to properly compare the low frequencies dynamics of the simulated and the measured pressure fluctuation signals, the Hilbert-Huang Transform Method, HHTM, is used to extract the information below the natural bed frequency. Thus, once the original time series have been decomposed into a finite number, $n$, of intrinsic mode functions, IMFs, associated with various time scales and the residual $r_{n}$, the time series of interest can be reconstructed as a surrogate of the original time series by superposition of the IMFs, $C_{n}(t)$, and the residual up to the bulk dynamic level as:

$$
x(t)=\sum_{j=1}^{n=b u l k} C_{j}(t)+r_{n}
$$

where $C_{\text {bulk }}(t)$ corresponds to the global bed motion caused by the gravitational oscillations of the fluidized bed material, which is characterized by the natural bed frequency.

Table 2 shows the averaged instantaneous frequencies estimated according to Briongos et al. (2006b), for the five modes corresponding to the simulated and measured MBR extracted through the EMD process. The stopping criterion has been set by the way in which the sifting process is stopped when the averaged instantaneous frequency of the current mode is smaller than the characteristic bubble exploding frequency $(0.5 \mathrm{~Hz})$. According to that, the mode $\left\langle w_{5}\right\rangle$ of Table 2 is the lower dynamical level drawn from the analysis.

As stated before, once the EMD process terminates, a "low-pass" filtered version, $x_{f}$, of both the simulated and the measured time series is reconstructed following Eq. 28 by superposition of the IMFs and the residual up to the bulk dynamic component. In order to estimate the natural bed frequency, $f_{b u l k}$, a conventional expression previously reported in literature such as that of Baskakov al (1986), $f_{\text {bulk }}=1.5 \mathrm{~Hz}$, might be used, however in this case it is concluded from Figure 5 that the model proposed by Roy et al. (1990) almost matches the natural bed frequency, $f_{\text {bulk }}=1.1 \mathrm{~Hz}$. Consequently, the bulk dynamic component is the one which has the average instantaneous frequency closest to $f_{\text {bulk }}$ (Table 2, bold frequencies).

The Figure 8 compares the temporal structure of the measured and the simulated surrogate time series by means of the power spectra, and the mutual information function. As for the case of SBR the power spectra is very similar, being the model 
spectra slightly shifted towards lower frequencies (Figure 8a). Moreover, the mutual information analysis matches, pointing out a high degree of dynamical similarity between the measured and the simulated dynamics (Figure 8b). Concluding with the dynamical comparison, the results from the state space analysis confirm the high degree of similarity between the compared dynamics (Figure 9). Thus, the reconstructed attractor, eigenvalue spectra, and attractor properties such as the correlation dimension and Kolmogorov entropy almost match. Therefore it is concluded that the simulated time series preserves both the linear and the nonlinear features of the measured dynamics.

In contrast to the SBR and MBR, the experimental evidence shows that ER occurs at gas velocities that are several times as high as the terminal velocity of the averaged bed particle size (Svensson et al. 1996a, b), under such fluidization conditions, the bottom bed dynamics is characterized by large irregular voids that promote a vigorous interaction with the dense phase. The Figure 10a shows, the power spectra of the simulated pressure time series. It can be observed that it is not just a shifted delay version of the experimental run, as was observed in the case of the MBR, now the power spectra for both surrogates of the simulated and the measured pressure time series are different. Moreover, as for the SBR case, the mutual information function, MIF, exhibits differences within the short-term temporal structure (Figure 10b). Thus at short-term scale, the MIF of the measured dynamics exhibits a minimum around $0.3 \mathrm{~s}$ whereas the simulated time series does not show any singular point and instead of that, its MIF smoothly decreases reaching its long-term memory value, which matches that of the measured dynamics. According to that, as for the SBR, the similarities within the long-term temporal structure are confirmed through the state space analysis. Thus when comparing the reconstructed attractors for both surrogates, it can be observed how the simulated PCA projection mimics both the shape and size of the core of the experimental reconstructed attractor (Figure 11). The slight differences between both projections, which are mainly due to the absence of outer orbits around the core of the reconstructed attractors from simulated signal, is explained by differences exhibited within the short-term temporal structure (Figure 10b), which comes from high frequency dense phase phenomena that are not taken into account by the model (Figure 10a). Moreover, the values of the Kolmogorov entropy and correlation dimension of the simulated pressure time series are very close to those of the measured signal.

Finally, in agreement with the results reported in literature (Svensson et al. 1996a,b), the simulated exploding bubble dynamics is independent of the distributor pressure drop, consequently, similar results are obtained when comparing simulated time series obtained for ER conditions by using different distributor pressure drops (Figure 12).

\section{Tuyere plate distributor}

Since perforated plate distributors cannot be used under severe operating conditions, other designs such as Tuyere plate distributors are used in those situations, therefore, due to the fact that many industrial processes use those distributor types, it is worth testing the reliability of the proposed approach to deal with the modeling of bubbling FB's operating with Tuyere plate distributors. Accordingly, as an example of the use of the proposed model for Tuyere type systems, the performance of a bench-scale combustor operating at ambient temperature is presented for model validation under current bubbling operating conditions (Table 1).

The validation of the proposed model for dynamic diagnosis and design of gas-solid fluidized beds having a Tuyere plate distributor, follows the same methodology previously used for MBR and ER analysis. Thus, the power spectra of both the 
measured and the simulated time series are shown in Figure 13a. Let us note that due to the fact that the pressure sensor used to monitor the bench-scale combustor is less sensitive than the Kistler pressure transducer, the direct comparison of the signals in the frequency domain reflects a high degree of similarity in MBR. However, since the MBR is characterized by an intense dynamical interaction between the bubble and the dense phase which is not taken into account by the model, the same multiscale approach as in the case of multiorifice distributor is used here for a detailed comparison when operating at MBR. Consequently, the HHTM is used to extract the information below the natural bed frequency. Subsequently, surrogates of the original time series are reconstructed by superposition of the IMF's and the residual up to the bulk dynamic level. It is clear from Figure 13b that as same as in the multi-orifice distributor case, the power spectra of the compared surrogates almost match, moreover the results from the MIF analysis confirm that both time series have a similar temporal structure, therefore, as expected, the subsequent state space analysis match, indeed it can be seen on Figure 14 how the attractor structure as well as its invariants remain the same for both the simulated and experimental data, which confirms the reliability of the model also for Tuyere type systems.

\section{Conclusions}

The proposed bubble-cap model operated either with the synchronous bubble generation mechanism (user defined overall bubble generation frequency), or with the asynchronous bubble generation model (distributor performance correction), matches the dynamic characteristics of bubble dynamics found on gas-solid fluidized beds, operating either at multiple or at single bubbling regimes, with gas velocities ranging between $2<U_{r}<6$, or at large fluidization velocities when the bed operates at exploding regime. Therefore the model is a suitable tool to be used to help in the dynamic diagnosis of gas-solid fluidized beds.

The activation region mechanism, resulting from the asynchronous bubble generation, makes it possible that the model accounts for the effect of the distributor performance due to the influence of the pressure drop across the distributor plate on bed regimes. Moreover, the model serves to simulate gas-solid fluidized beds having either multiorifice or Tuyere type distributors, being a useful tool to be applied to help in the design and operation of bubbling fluidized beds.

Through the multiscale nonlinear approach used for model validation, some dynamical aspect characteristics of bubbling dynamics such as exploding bubble phenomena have been identified by establishing a direct relation between the physical phenomena driving the dynamics (bubble generation, interaction and eruption), and the measured signals. Moreover, the simplicity of the model assumptions facilitates the understanding of the dynamics behind the measured signals, and how the different signal properties obtained from the time series analysis are related to the physical phenomena occurring within the FB's; in that sense Figure 11 is a beautiful example of that.

Finally, though the results are very promising, the model has been tested only with cold bed installation and more research is needed to get an insight into multi-orifice bubble distribution and hot bed facilities.

\section{Notation}
$A_{\text {bed }} \quad$ bed area, $m^{2}$
$C_{j} \quad$ IMF component
$C_{v} \quad$ virtual mass force coefficient
$\mathrm{D}_{2} \quad$ correlation dimension, [-] 
$d_{b 0} \quad$ initial bubble size from synch-approach, $m$

$d_{b} \quad$ bubble diameter, $m$

$d_{L b} \quad$ leading bubble diameter, $m$

$E \quad$ power spectrum energy, [-]

ev normalized eigenvalue (Daw and Halow, 1993), [-]

$f_{a} \quad$ fraction of active area, [-]

$f_{b} \quad$ overall bubble generation frequency, $\mathrm{Hz}$

$f_{b, o r} \quad$ bubble orifice frequency, $\mathrm{Hz}$

$f_{w} \quad$ wake fraction, [-]

$G \quad$ bubble flow per hole, $\mathrm{m}^{3} / \mathrm{s}$

$h_{f} \quad$ bed height, $m$

I mutual information function,[bits]

$K_{c} \quad$ Kolmogorov entropy per cycle, [bits/cycle]

$m$ dimensionless embedding dimension, [-]

$\mathrm{N}_{\mathrm{b}} \quad$ Number of bubbles in the histogram

$N_{o r} \quad$ Number of orifices, [-]

$p_{R} \quad$ pressure recovery, $\mathrm{Pa}$

$Q_{c} \quad$ gas transfer rate during coalescence, $\mathrm{m}^{3} / \mathrm{s}$

$R_{b} \quad$ bubble radius, $m$

$r \quad$ bubble to pressure probe distance, $m$

$r_{n} \quad$ decomposition residual

$t_{d} \quad$ bubble detachment time, $s$

$U_{b} \quad$ bubble velocity, $\mathrm{m} / \mathrm{s}$

$U_{i b} \quad$ isolation bubble velocity, $\mathrm{m} / \mathrm{s}$

$U_{0} \quad$ superficial gas velocity, $\mathrm{m} / \mathrm{s}$

$U_{m f} \quad$ minimum fluidization velocity, $\mathrm{m} / \mathrm{s}$

$U_{t b} \quad$ trailing bubble velocity, $\mathrm{m} / \mathrm{s}$

$U_{\text {void }}$ void propagation velocity, $\mathrm{m} / \mathrm{s}$

$u_{b, o r} \quad$ initial bubble velocity from synch-approach, $\mathrm{m} / \mathrm{s}$

$u_{o r} \quad$ gas velocity through the orifice, $\mathrm{m} / \mathrm{s}$

$V_{b} \quad$ visible bubble flow, $\mathrm{m}^{3} / \mathrm{s}$

$v_{b} \quad$ bubble size of active region, $m^{3}$

Greek letters

$\alpha_{b} \quad$ stirring factor due to bubbles. [-]

$\alpha_{D, a} \quad$ stirring capacity of the active area region, [-]

$\alpha_{j} \quad$ stirring factor due to jets, [-]

$\varepsilon_{f} \quad$ void fraction, [-]

$\phi \quad$ deviation coefficient from synch-approach, [-]

$\theta \quad$ angle between vector from origin to pressure probe tip and the positive vertical axis

$\rho_{g} \quad$ gas density, $\mathrm{kg} / \mathrm{m}^{3}$

$\rho_{e} \quad$ emulsion density, $\mathrm{kg} / \mathrm{m}^{3}$

$\rho_{p} \quad$ particle density, $\mathrm{kg} / \mathrm{m}^{3}$

$\psi \quad$ dimensionless ratio between observed bubble flow and the excess flow, [-]

Abbreviations

$\mathrm{CBF} \quad$ circulating fluidized bed

EMD empirical mode decomposition

ER exploiding bubble regime

HHTM Hilbert-Huang transform method

IMF intrinsic mode function 
MBR multiple bubble regime

MIF mutual information function analysis

PCA principal component analysis

SBR single bubble regime

\section{Acknowledgement}

The author would like to specially thank Prof. Filip Johnsson (Chalmers University) who kindly provided the Kistler pressure time series for model validation.

\section{Appendix A. On Multi-orifice injection Pattern}

According to Figure 1 it is clear that the bubble injection pattern has some influence on the bubble structure developed within the bed. Thus, it can be seen that when using injection patterns such as those of Figure 1a, b, which represent an extreme situation, where there are "inactive" regions on the distributor plate, wherever the bubble generation is negligible compared to the actives zones, the resulting bubble dynamical structure is strongly influenced by the bubble generation. That fact is enhanced within the proposed approach due to the fact that the XY displacement is motivated by the bubble-bubble interaction. However, when the FB system is fitted with a uniform gas distributor (Figure 1c), the resulting bubble pattern is not homogeneous and instead of promoting a Type 2 system (Whitehead, 1985), the resulting pattern can be identified as a Type 1. Accordingly, the pattern is exhibiting preferred paths for rising bubbles and descending solids.

In order to identify the optimum bubble injection pattern that leads to a dynamical matching between the simulated and measured dynamics, the power spectra of the different bubble generation situations are shown on Figure A1. When comparing the simulated results to the measured pressure time series, Figure A1c that corresponds to a uniform bubble generation pattern, is qualitatively and quantitatively very similar to the measured pressure time series. Consequently, the bubble pattern existing within the cold rig under evaluation is identified as a Type 1 system. Once the bubble structure has been identified as Type 1, the bubble generation pattern can be modified facilitating the solid preferred circulation paths (Figure 1d), and improving the matching between the simulated and the measured dynamics (Figure A1d). The state space analysis can be used to help with the injection patter selection (Figure A2). From Figures A1 and A2 it is clear that using different bubble generation patterns is a method for applying the model for dynamical matching purposes. 


\section{Literature}

Caram H.S., Hsu K.-K. (1986). Bubble formation and gas leakage in fluidized beds. Chemical Engineering Science 41, 1445-1453.

Cui H., Grace J.R. (2007). Fluidization of biomass particles: A review of experimental multiphase flow aspects. Chemical Engineering Science 62, 45-55.

Baskakov, P., Tuponov, V.G., Filippovsky, N.F., 1986. A study of pressure fluctuations in a bubbling fluidized bed. Powder Technology 45, 113-117.

Bokkers G.A., Laverman J.A., van Sint Annaland M., Kuipers J.A.M. (2006). Modelling of large-sclae dense gas-solid bubbling fluidised beds using a novel discrete bubble model. Chemical Engineering Science 61, 5590-5602.

Briongos J.V., Guardiola J. (2003). "Free top fluidized bed surface fluctuations as a source of hydrodynamic data". Powder Technol. 134, 133-144.

Briongos J.V., Aragón J.M., Palancar, M.C. (2006a) . Fluidised bed dynamics diagnosis from measurements of low frequency out bed passive acoustic emissions. Powder Technol. 162, 145-156.

Briongos, J.V., Aragón, J.M. Palancar, M.C. (2006b). "Phase space structure and multiresolution analysis of gas solid fluidized beds hydrodynamics: Part I: The EMD approach". Chem. Eng. Sci. 61, 6963-6980.

Briongos, J.V., Aragón, J.M. Palancar, M.C. (2007). "Phase space structure and multiresolution analysis of gas solid fluidized beds hydrodynamics: Part II: Dynamic analysis". Chem. Eng. Sci. 62, 2865-2879.

Broomhead, D.S. y King, G. P. (1986). Qualitative dynamics from experimental data, Physica D, 20, 217-236.

Clift R., Grace J.R. (1971). Coalescence of Bubbles in fluidized beds. AIChE Symp. Ser. 116 vol. $67,23-33$.

Daw C.S. and Halow J.S. (1992). Modelling deterministic chaos in gas fluidized beds. AIChE Symp. Ser. 289 vol. 88, 61-69.

Daw, C. S. y Hallow, J. S. (1993). Evaluation and control of fluidization quality through chaotic time series analysis of pressure drop measurements, AIChE Symp. Ser., 89, (296), 103-122.

Davidson J.F,, Clift R., Harrison D. (1985). Fluidization (2nd Ed.). Academic Press Inc. (London). ISBN: 0-12-205552-7.

Davidson J.F., Harrison D. (1963). Fluidised particles. Cambridge University Press. 
Deen N.G., van Sint Annaland M., van der Hoef M.A., Kuipers J.A.M. (2007). Review of discrete particle modeling of fluidized beds. Chemical Engineering Science 62, 2844 .

Ding J., Gidaspow D. (1991). A bubbling fluidization model using kinetic theory of granular flow. AIChE J. 36, 523-538.

Dyakowski, T., Jeanmeure, L.F.C., Jaworski, A.J. (2000). Applications of electrical tomography for gas-solids and liquid-solids flows - A review, Powder Technol. 112, 174-192.

Fiorentino F., Marzocchela A. and Salatino P. (1997). Segregation of fuel particles and volatile matter during devolatilization in a fluidized bed reactor -I. Model development. Chemical Engineering Science 52, 1909-1922.

Fraser, A. M. and Swinney, L. H. (1986). Independent coordinates for strange attractors from mutual information, Phys. Rev. A., 33, 1134-1140.

Gidaspow D. (1994). Multiphase flow and fluidization. Academic Press Inc. ISBN: 012-282470-9.

Grassberger, P., Procaccia, I., 1983. Characterization of strange attractors. Physical Review Letters 50, 346-349.

Grace J.R., Harrison D. (1969). The behavior of freely bubbling fluidised beds. Chemical Engineering Science 24, 497-508.

Hoffmann A.C., Janssen L.P.B.M., Prins J. (1993). Particle segregation in fluidized binary mixtures. Chemical Engineering Science 48, 1583-1592.

Johnsson F., Andersson S., Leckner B. (1991). Expansion of a freely bubbling fluidized bed. Powder Technology 68,117-123.

Johnsson, F., Zjierveld, R.C., Schouten J.C., van den Bleek, C.M., 2000. Characterization of fluidization regimes by time-series analysis of pressure fluctuations. International Journal of Multiphase. Flow 26, 663-715.

Jonhsson F. (2007). Fluidized Bed Combustion for Clean Energy, Conference on the 12th International Conference on Fluidization - New Horizons in Fluidization Engineering. Paper \#5 , pg. 47 - 62. ISBN 978-0-918902-57-3.

Kendoush A.A. (2003). The virtual mass of a spherical-cap bubble.. Physics of fluids $15,2782-2785$.

Kunii D., Levenspiel O. (1991). Fluidization Engineering. Butterworth-Heinemann. ISBN: 0-409-90233-0.

Leung L.S. (1971). Design gas distributor and prediction of bubble size in large GasSolids Fluidized beds. Powder Technology 6, 189-193. 
Lindborg H., Lysberg M., Jakobsen H.A. (2007). Practical validation of the two-fluid model applied to dense gas-solid flows in fluidized beds. Chemical Engineering Science 62, 5854-5869.

Lockett M.J., Davidson J. F., Harrison D. (1967). On the two-phase theory of fluidization. Chemical Engineering Science 22, 1059-1066.

Müller C.R., Holland D.J., Davidson J.F., Dennis J.S., Gladden L.F., Hayhurst A.N., Mantle M.D. and Sederman A.J. Geometrical and hydrodynamical study of gas jets in packed and fluidized beds using magnetic resonance. The Canadian Journal of Chemical Engineering 87, 517-525.

Pannala S., Daw C.S., Halow J.S. (2004). Dynamic interacting bubble simulation (DIBS): an agent-based bubble model for reacting fluidized beds. Chaos 14, 487-498.

Rees A.C., Davidson J.F., Dennis J.S., Fennell P. S, Gladden L.F., Hayhurst A.N., Mantle M.D., Müller C.R., Sederman A.J. The nature of the flowjust above the perforated plate distributor of a gas-fluidised bed, as imaged using magnetic resonance. Chemical Engineering Science 61, 6002 - 6015.

Roy, R., Davidson, J.F., Tuponogov, V.G., 1990. The velocity of sound in fluidised beds. Chemical Engineering Science 45, 3233-3245

Schouten, J.C., Takens, F., van den Bleek, C.M., 1994. Maximum likelihood estimation of the entropy of an attractor. Physical Review E 49, 126-129.

Ruzicka M., Drahos J., Zahradnik J., Thomas N. H. Natural modes of multi-ori"ce bubbling from a common plenum. Chemical Engineering Science 54, 5223-5229.

Schouten, J.C., Takens, F. y Van den Bleek, C. M. (1994b). Maximun likelihood estimation of the entropy of an atractor, Phys. Rev. E, 49, 126-129.

Svensson A., Johnsson F., Leckner B. (1996a). Fluidization regimes in non-slugging fluidized beds: the influence of pressure drop across the air distributor. Powder Technology 86, 299-312.

Svensson A., Johnsson F., Leckner B. (1996b). Bottom bed regimes in a circulating fluidized bed boiler. Int. J. Multiphase flow 22, 1187-1204.

Tsuji T., Yabumoto K., Tanaka T. (2008). Spontaneous structures in three-dimensional bubbling gas-fluidized bed by parallel DEM-CFD coupling simulation. Powder Technology 184, 132-140.

Vakhshouri K., Grace J.R. (2008). Modeling of bubble formation at a submerge orifice in gas-fluidized bed. Chemical Engineering Research and Design. Doi: 10.1016/j.cherd.2008.10.002.

Van Ommen J.R., Mudde R.F. (2007). "Measuring the Gas-Solids Distribution in Fluidized Beds - A Review". Conference on the 12th International Conference on Fluidization - New Horizons in Fluidization Engineering. Paper \#4 , pg. 31 - 46. ISBN 978-0-918902-57-3. 
Van Wachem B.G.M., Schouten, J.C., van den Bleek C.M., Krishna R., Sinclair J.L. (2001). Comparative analyisis of CFD models of dense gas-solid systems. AIChE J. 47, 1035-1051.

Van Wachem B.G.M., Schouten J.C., Krishna R., van den Bleeck C.M. (1999). Validation of the Eulerian simulated dynamic behaviour of gas-solid fluidized beds. Chemical Engineering Science 54, 2141-2149.

Van den Bleek, C.M., Schouten, J.C., 1993. Can deterministic chaos create order in a fluidized-bed scale-up, Chemical Engineering Science 48, 2367-2373.

Werther J. (1999). "Measurement techniques in fluidized beds", Powder Technol. 102, $15-36$.

Whitehead A.B. (1985). Distributor characteristics and bed properties, in: Fluidization $2^{\text {nd }}$ Ed. Academic Press. ISBN: 0-12-205552-7.

Zhang R., Tan R.B.H. (2000). A model for Hubble formation and weeping at a submerged orifice. Chemical Engineering Science 55, 6243-6250.

Xiao S., Tan R.B.H. (2003). Bubble formation at multiple orifices-bubble synchronicity and frequency. Chemical Engineering Science 60, 179-186. 


\section{Figure Caption}

Fig. 1. Bubble injection pattern, a, b, c, and d, and corresponding bubble dynamical structures developed within the bed, $-\mathrm{p}$, used during the simulations. The simulation correspond to SBR conditions having $\mathrm{N}_{\text {or }}=1660$ : $\mathrm{a}, \mathrm{b}$ ) injection patterns with inactive bubble generation regions; c) uniform gas distribution; d) modified uniform gas distribution to match Type 1 systems.

Fig. 2. Two-dimensional Fluidized bed operating at bubbling regime. The solid line accounts for the limit of the orifice interaction region, JR; the active regions i.e bubbles, AR, will appear above the JR area.

Fig. 3. Spherical-cap discrete bubble model: a) spherical cap, X-Y displacement is due to bubble interaction; b) Pressure recovery phenomena; c) Trailing effect.

Fig.4. Influence of pressure drop across the air distributor: a1, b1, c1, d1, e1) power spectra for distributor plates having respectively $\mathrm{N}_{\text {or }}=117,198,414,792,1660 ; \mathrm{a} 2, \mathrm{~b} 2$, c2, e2, d2) corresponding bubble patterns developed within the bed; a3, b3, c3, d3, e3) bubble size histogram.

Fig. 5. Comparison of the simulated signal with the measured Kistler pressure signal used in Jonhsson et al. (2000): a) Single bubble regime; b) Multiple bubble regime; c) Exploiding bubble regime.

Fig. 6. Mutual information function analysis for simulated and measured signal taken at SBR conditions. Note the short-term difference between experimental and simulated data.

Fig. 7 State space analysis of the experimental and the simulated pressure time series at SBR conditions: $a, b)$ reconstructed attractors; $c, d$ ) eigenvalue spectra.

Fig. 8. Time structure comparison for the surrogate signals obtained from the multiresolution analysis applied over the measured and the simulated pressure time series for multiple bubble regime: a) frequency domain comparison; b) mutual information function analysis.

Fig. 9. State space analysis for the surrogate signals for multiple bubble regime: a, b) reconstructed attractors; c, d) eigenvalue spectra.

Fig. 10. Time structure comparison for the surrogate signals for exploiding bubble regime: a) frequency domain comparison; b) mutual information function analysis.

Fig. 11. State space analysis for the surrogate signals for exploiding bubble regime: $a, b)$ reconstructed attractors; c, d) eigenvalue spectra.

Fig.12. Power spectra of simulated time series obtained for exploiding regime operating conditions having distributor plate with $\mathrm{N}_{\text {or }}=198,414,1660$ respectively.

Fig. 13. Time structure comparison for the Tuyere type system operating at multiple bubble regime: a) frequency domain comparison of the measured and simulated 
pressure signals ; b) power spectra of surrogate signals; c) mutual information function analysis over the surrogates.

Fig. 14. State space analysis for the surrogate signals of the Tuyere Type system at multiple bubble regime: $a, b)$ reconstructed attractors; c, d) eigenvalue spectra.

Fig. A1. Bubble injection pattern identification, frequency domain analysis. The simulated time series correspond to the patterns shown in Figure 1, SBR conditions, $\mathrm{N}_{\text {or }}$ = 1660: a) F1a-p system; b) F1b-p systems; c) F1c-p system (uniform); d) F1d-p system.

Fig. A2. Bubble injection pattern identification, state space analysis. The simulated time series correspond to the patterns shown in Figure 1, F1, SBR conditions, $N_{\text {or }}=1660$ : a) F1a-p system; b) F1b-p systems; c) F1c-p system (uniform); d) F1d-p system. 


\section{Table caption}

Table 1. Experimental condition used through the simulations.

Table 2. Averaged instantaneous frequencies for five modes corresponding to the simulated and measured time series extracted, at multiple bubble regime, through the EMD process. 


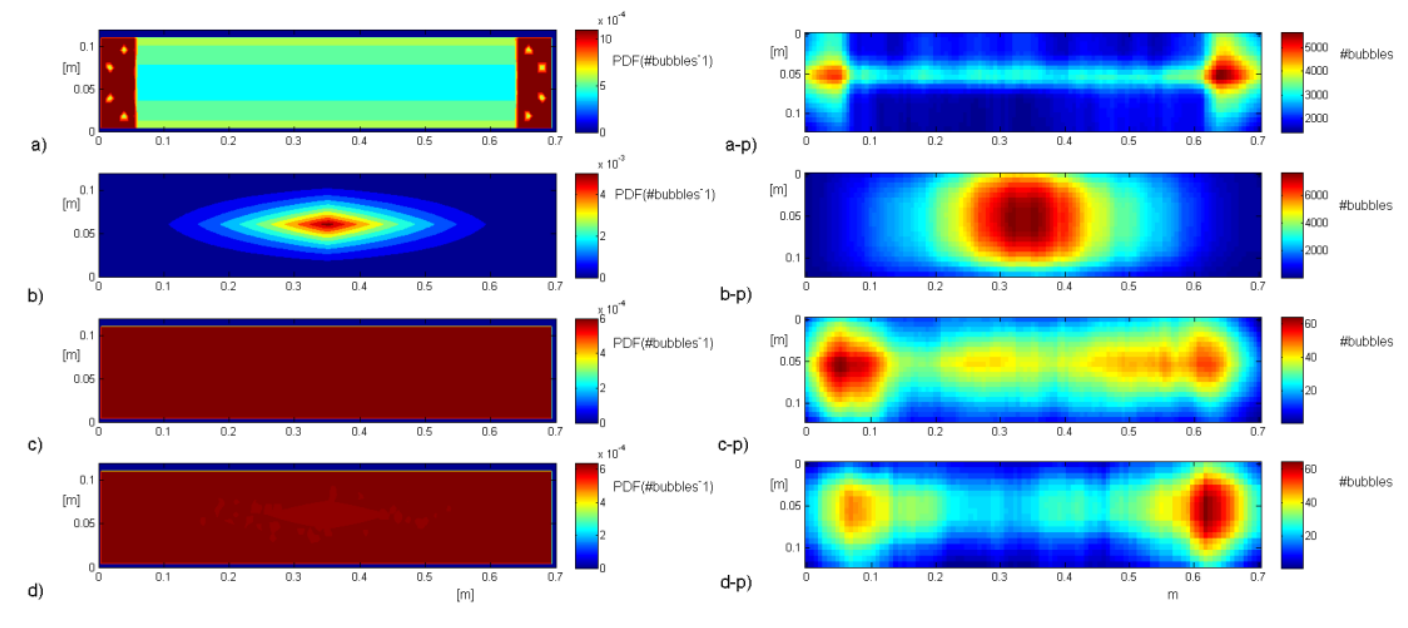

Figure 1

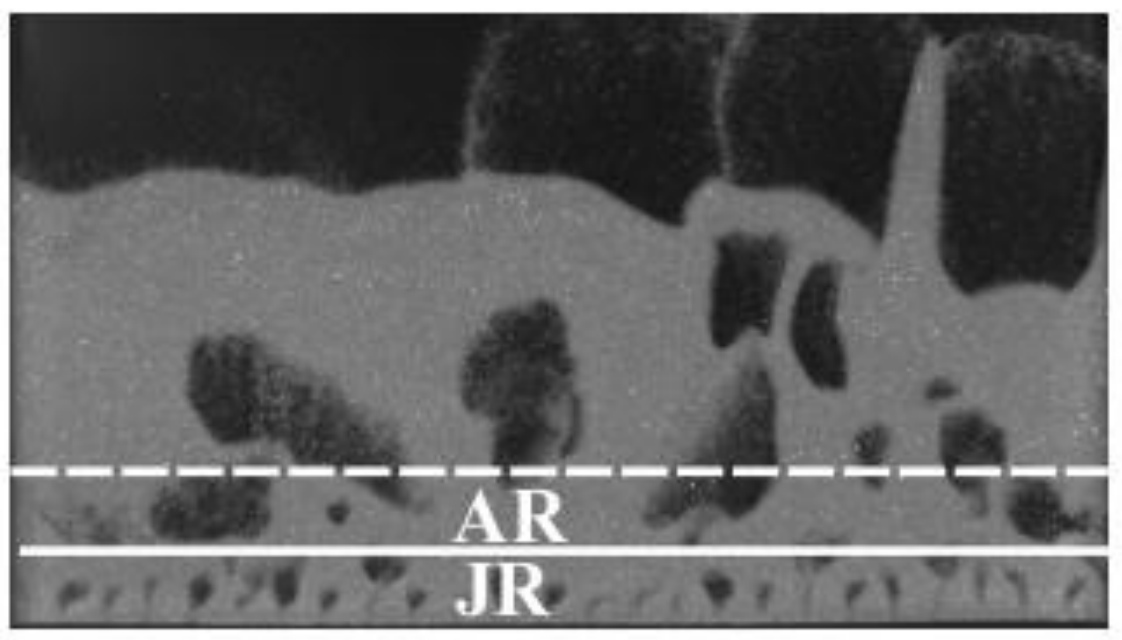

Figure 2 


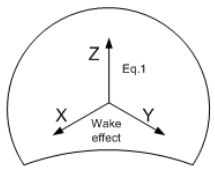

a

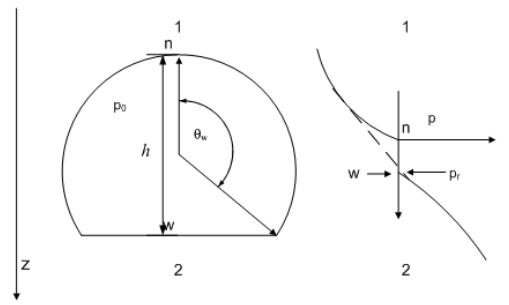

b

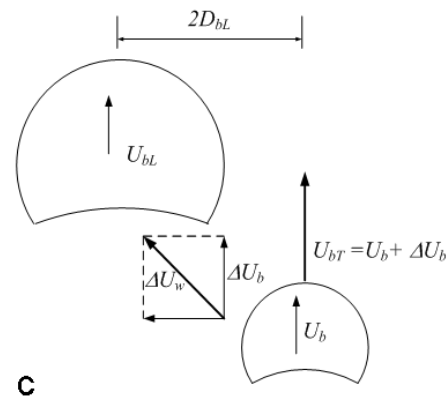

Figure 3

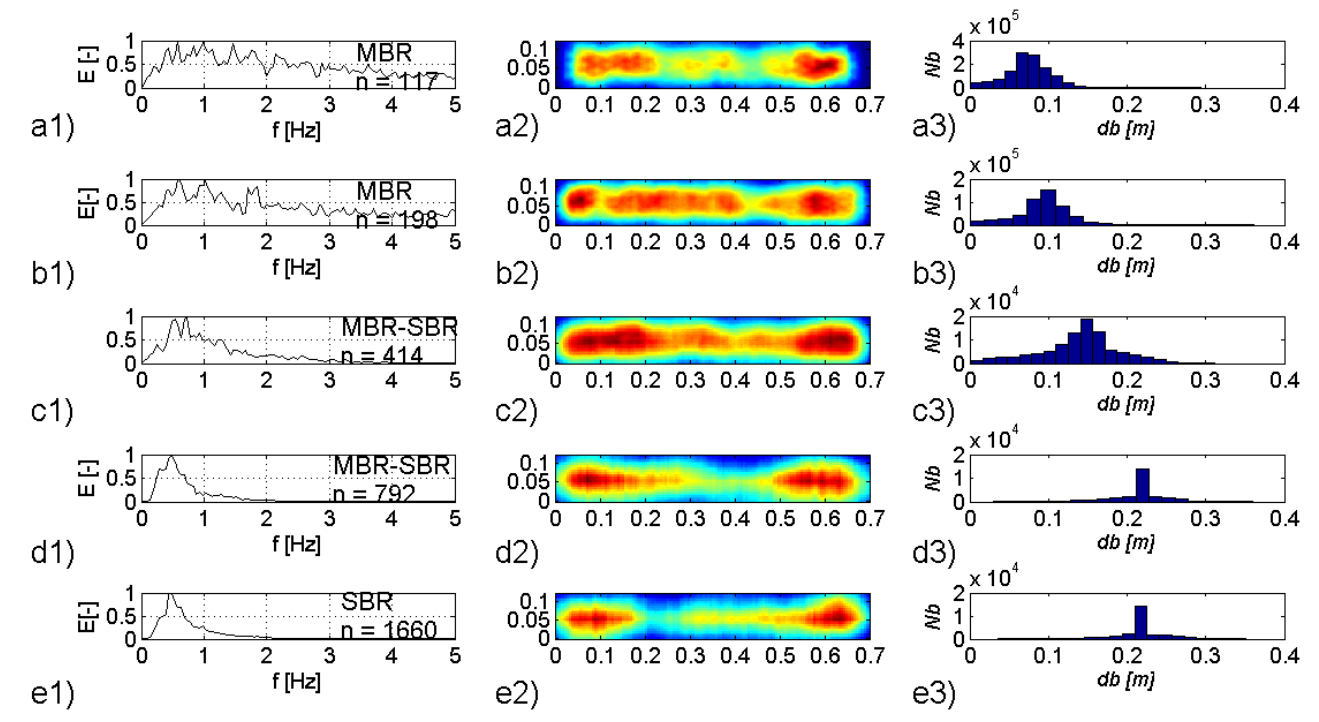

Figure 4 

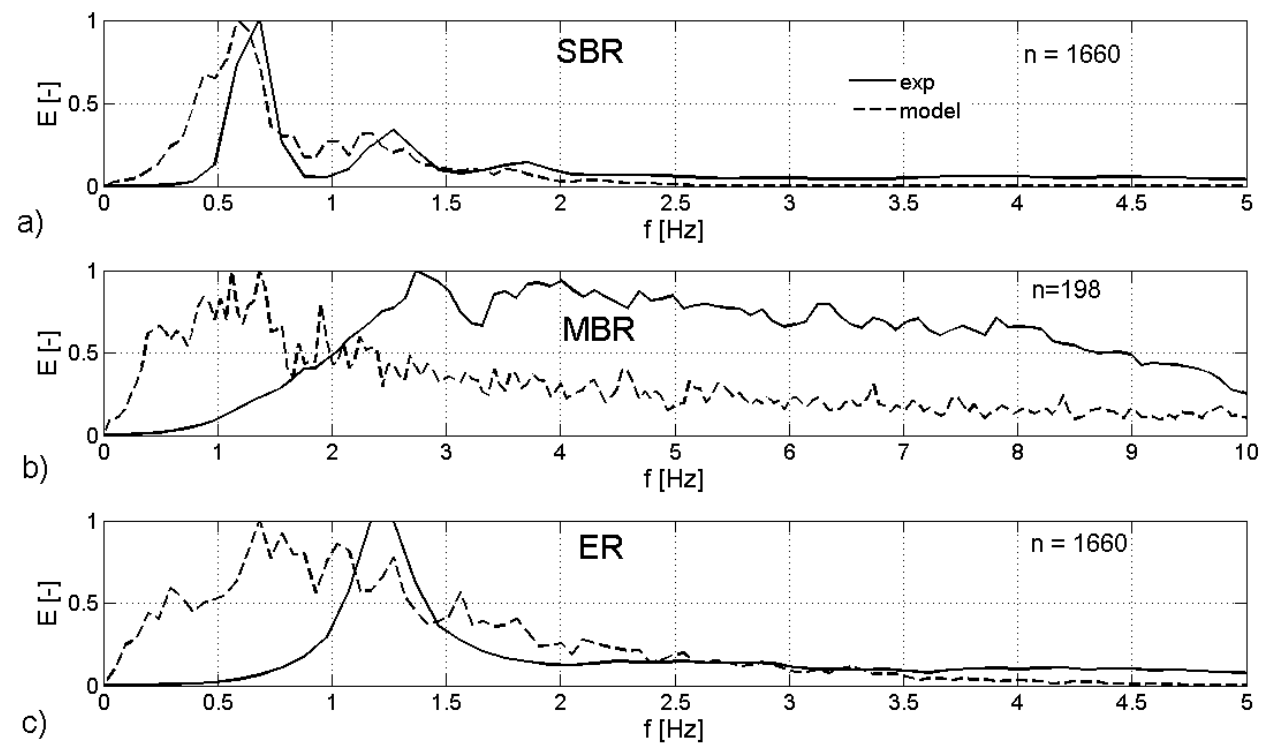

Figure 5 


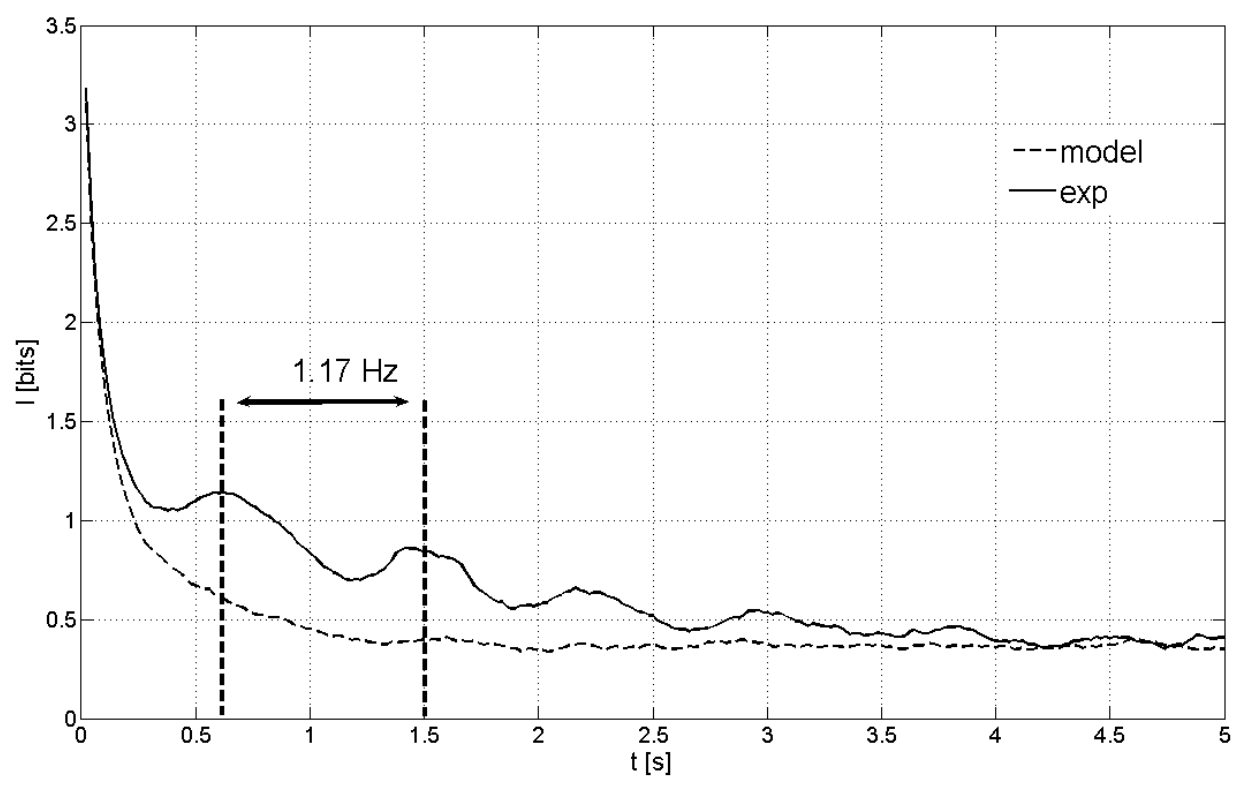

Figure 6 SBR

a)

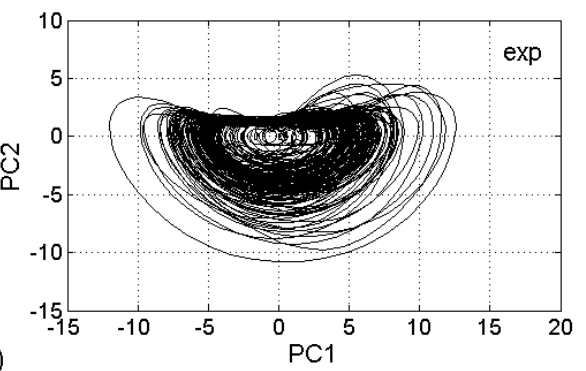

c)

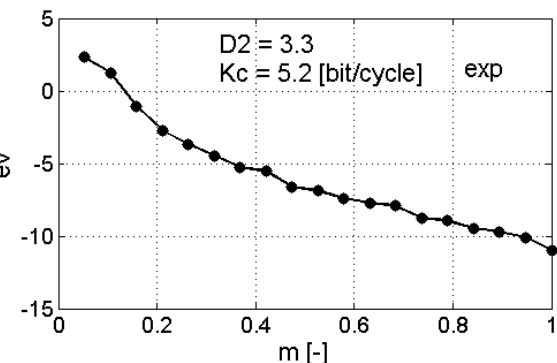

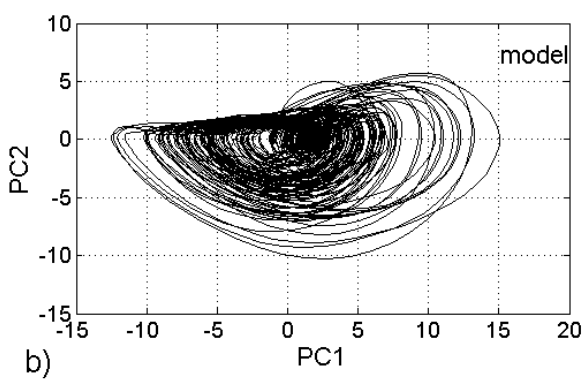

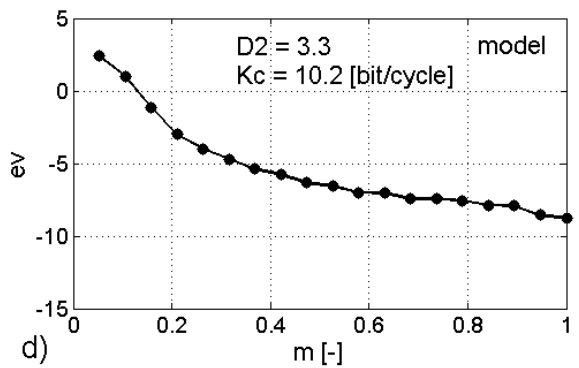

Figure 7. SBR 


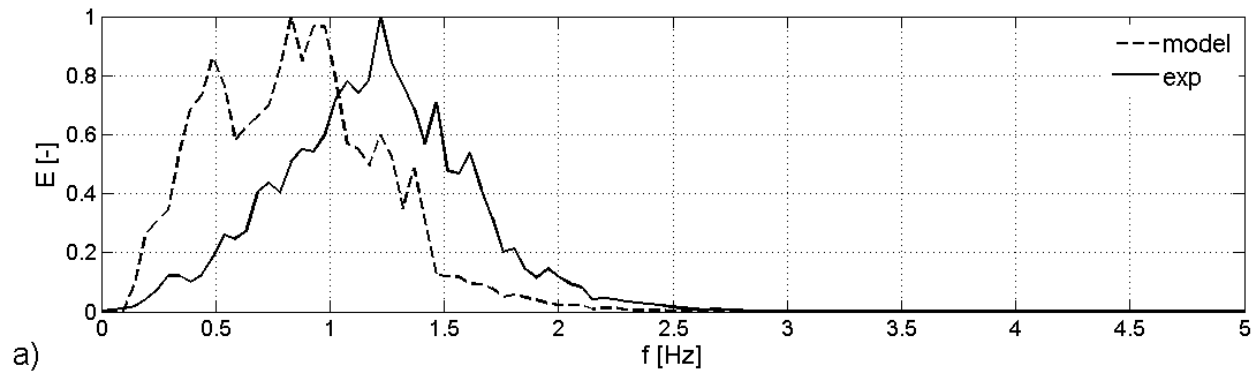

b)

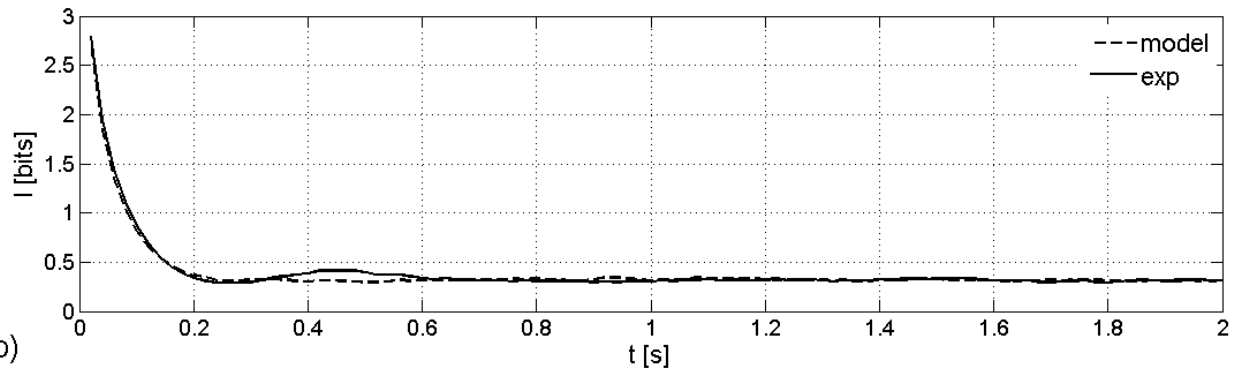

Figure $8 \mathrm{MBR}$

a)

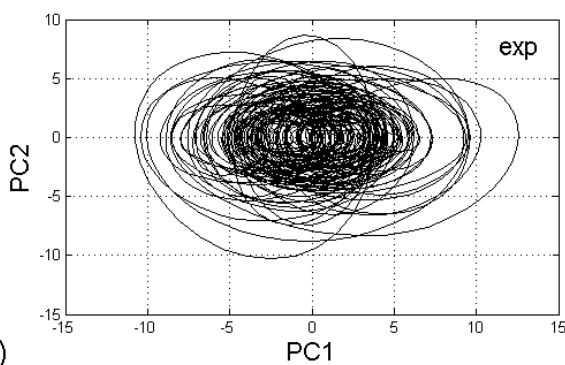

c)

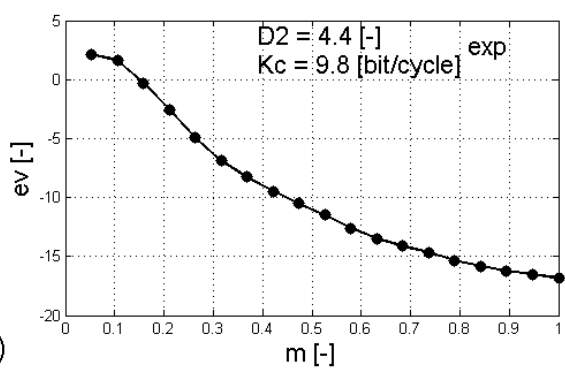

b)

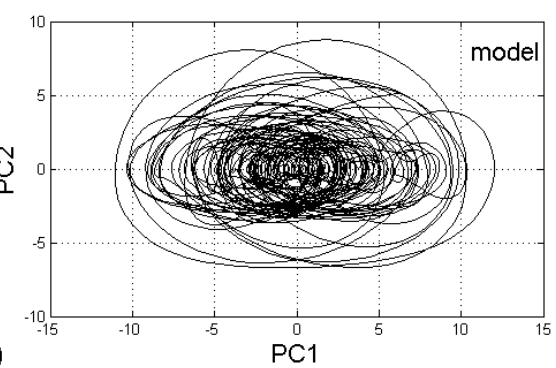

d)

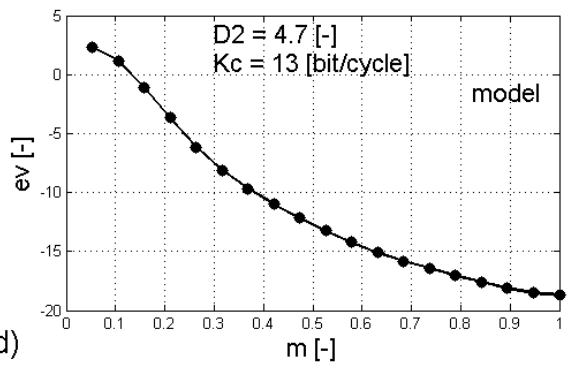

Figure 9 MBR 
a)

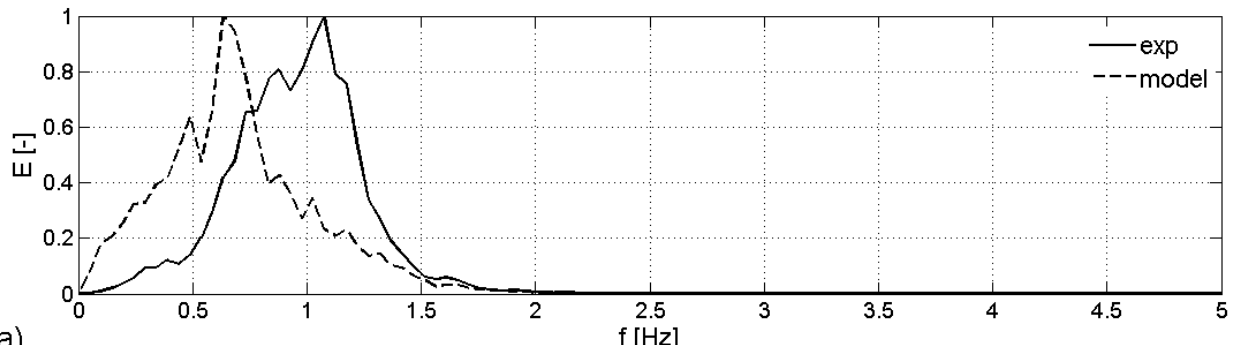

b)

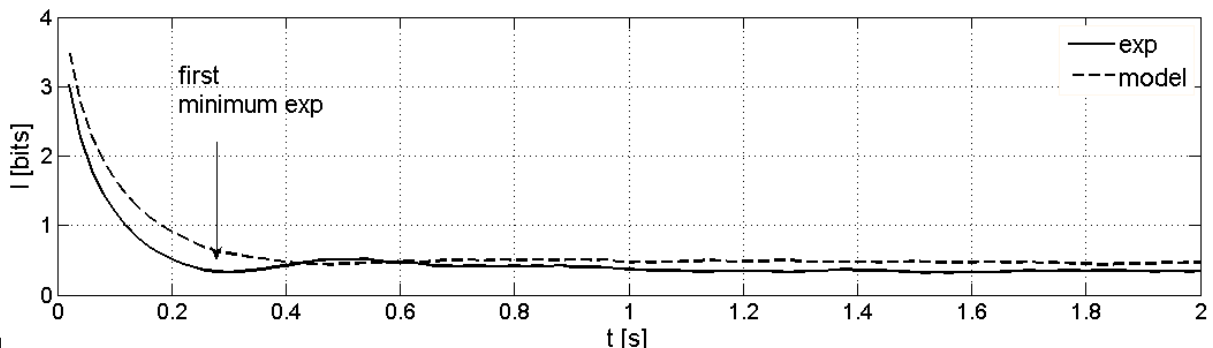

Figure 10 ER

a)

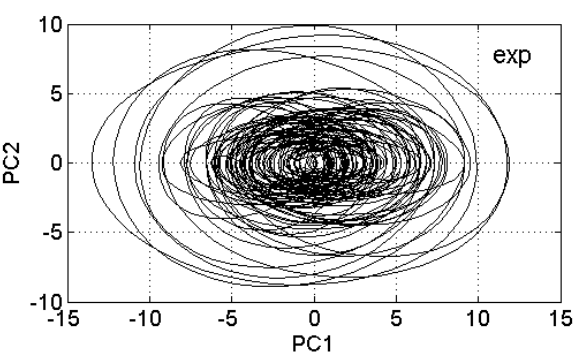

c)

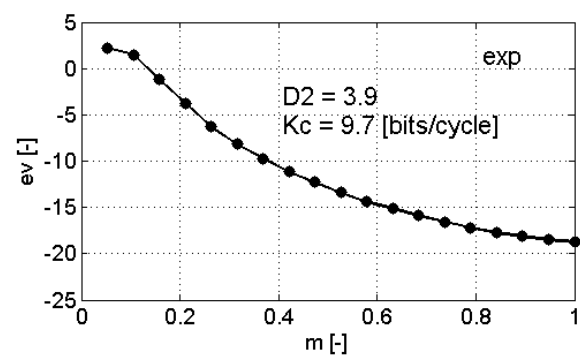

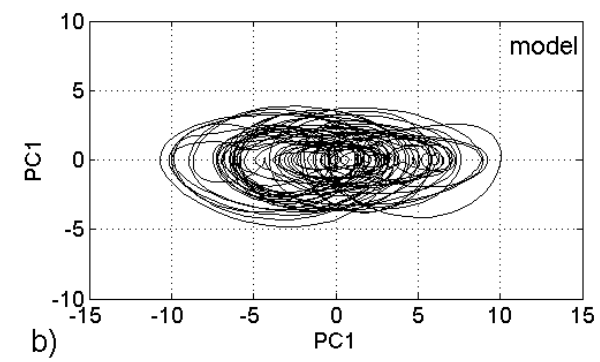

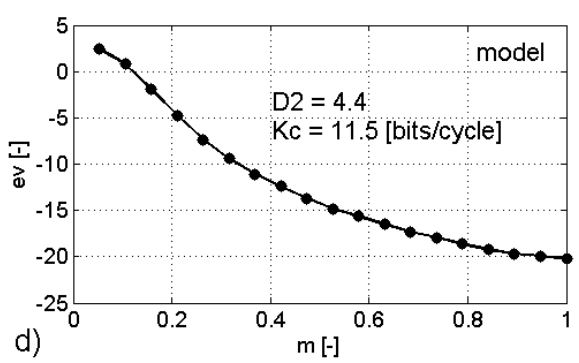

Figure 11 ER 


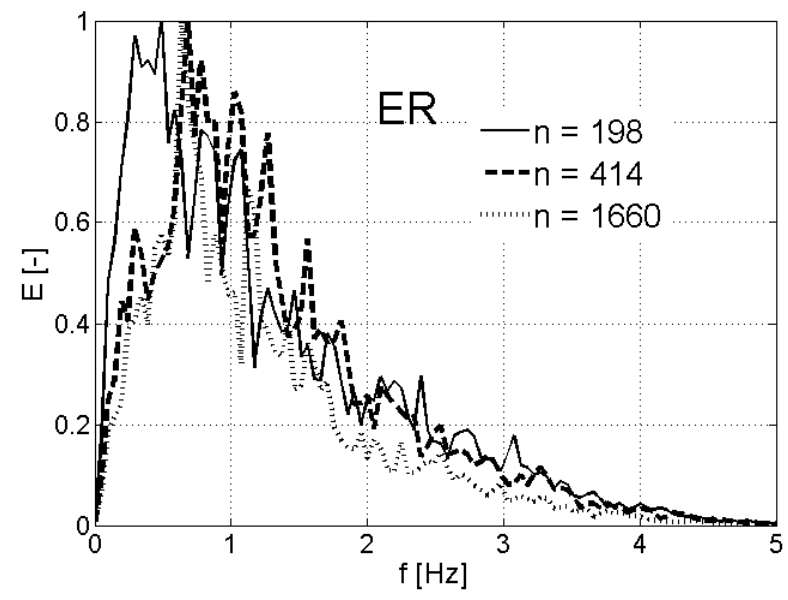

Figure 12

Tuyere Type
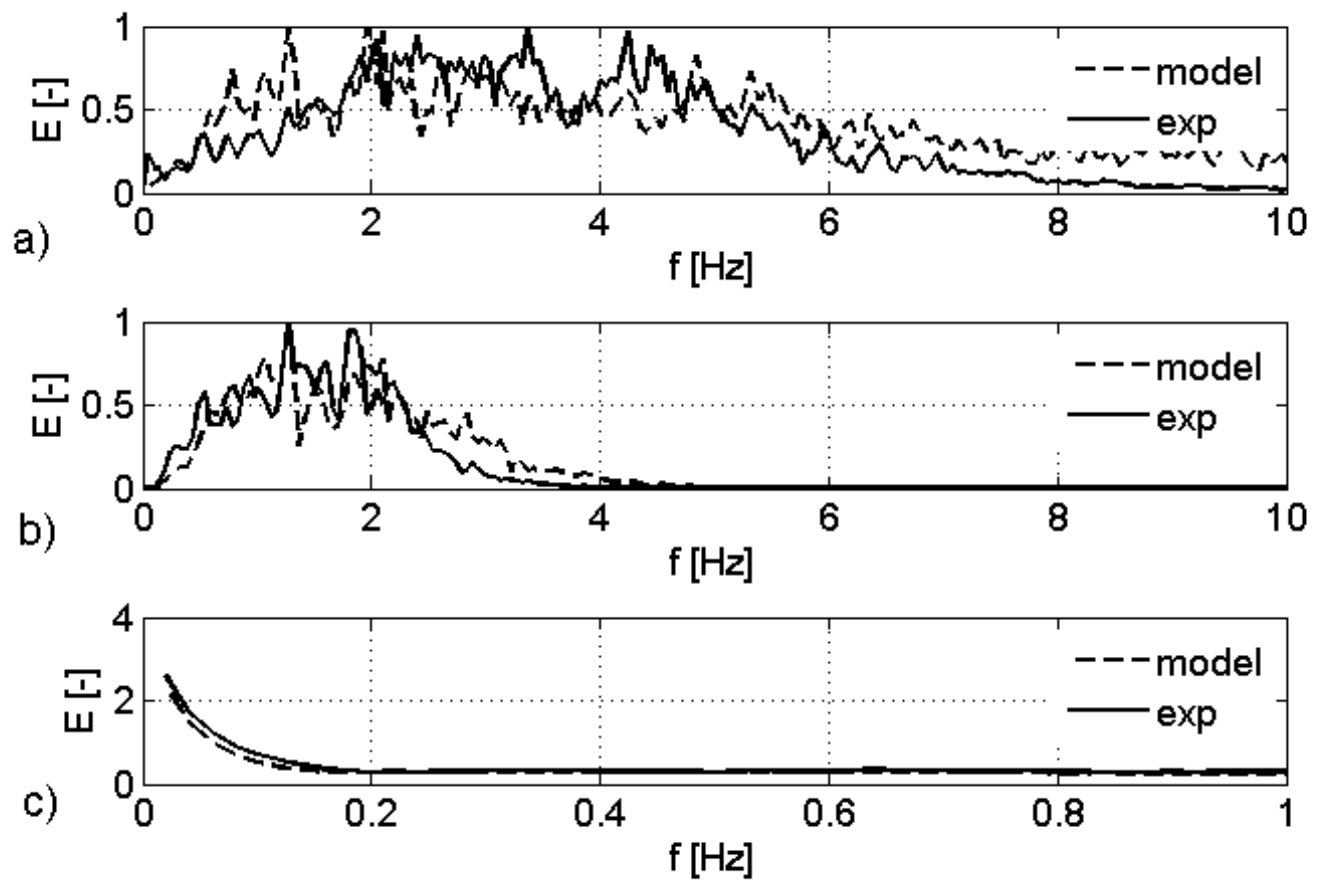

Figure 13 

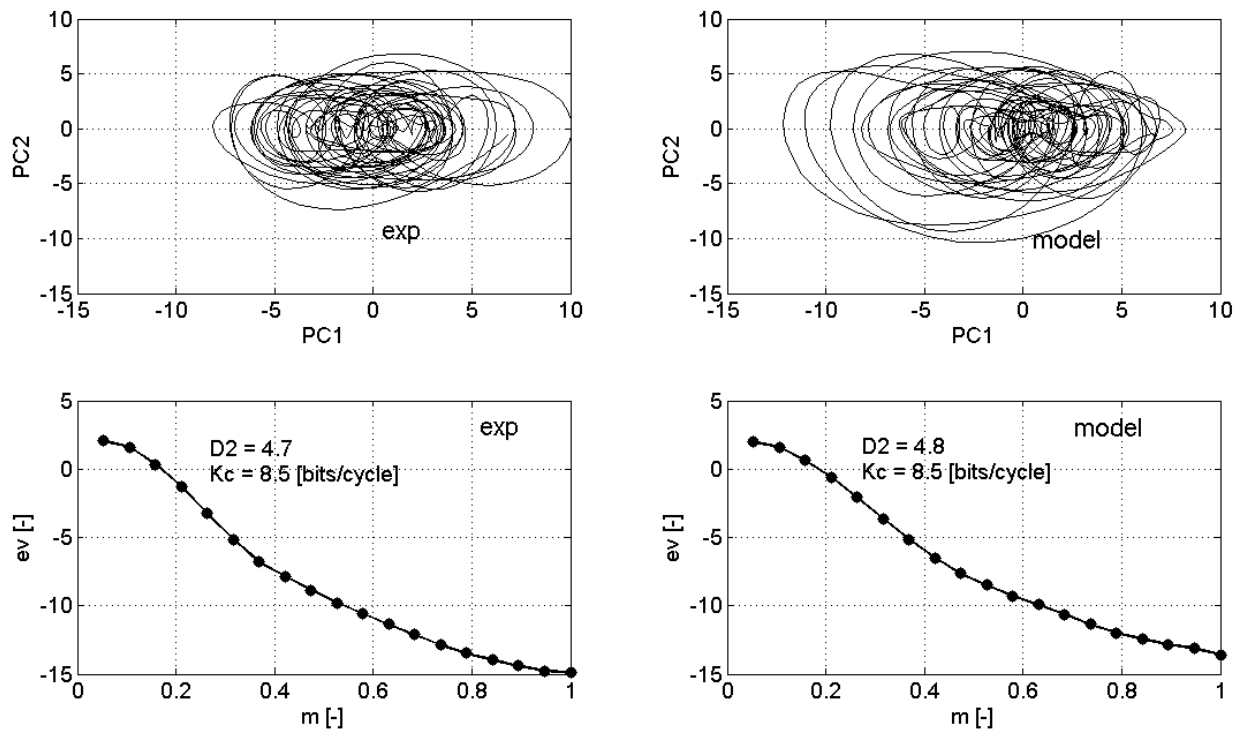

Figure 14

a)

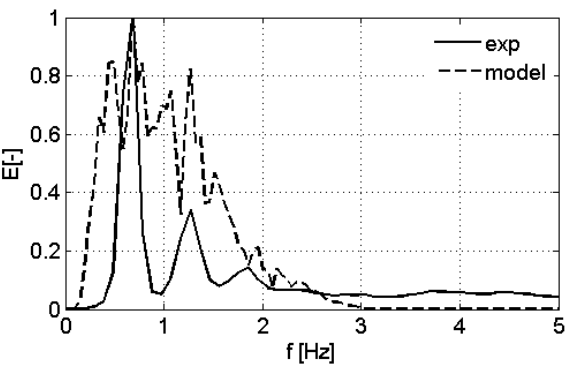

c)

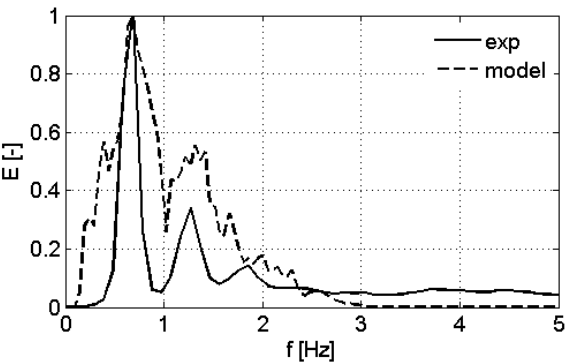

b)

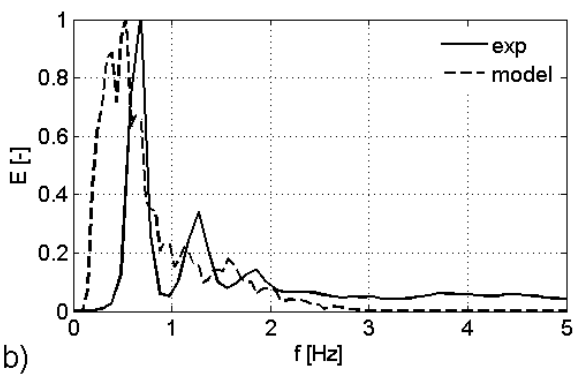

d)

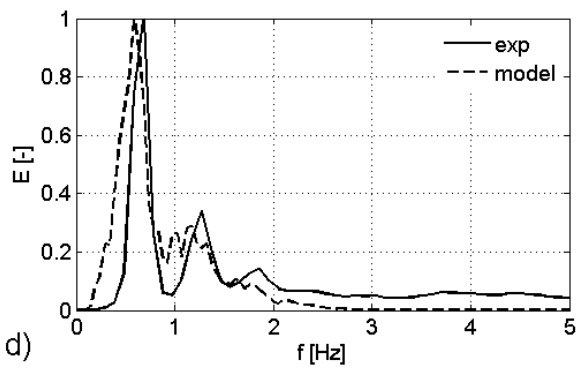

Figure A1 
a)

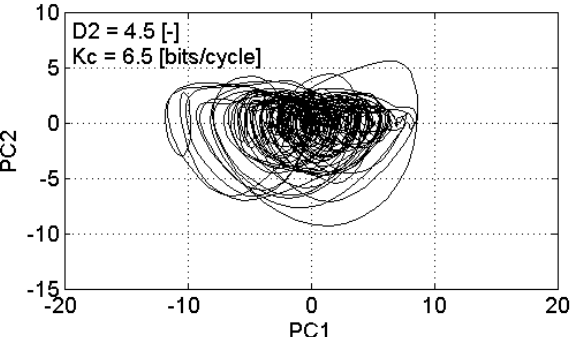

c)

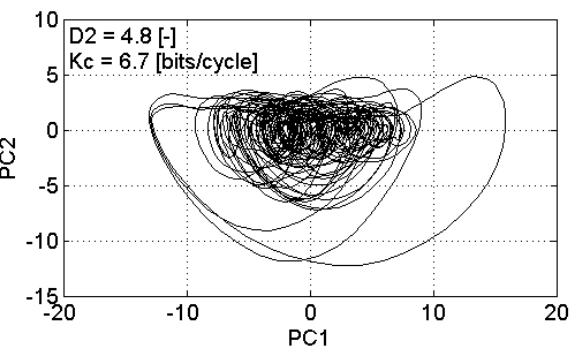

b)

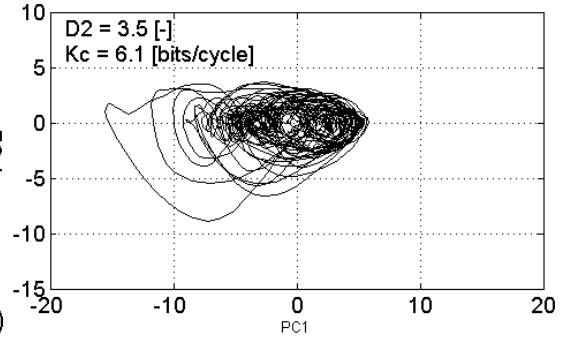

d)

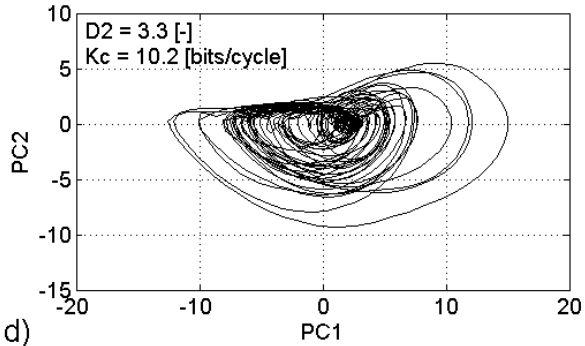

Figure A2 